\title{
Thermal Sensitivity and Cardiovascular Reactivity to Stress in Healthy Males
}

\author{
Pablo Antonio Conde-Guzón', María Teresa Bartolomé-Albistegui², \\ Pilar Quirós ${ }^{3}$, and Raúl Cabestrero ${ }^{3}$ \\ ${ }^{1}$ Universidad de León (Spain) \\ ${ }^{2}$ Hospital Nuestra Señora de Regla (Spain) \\ ${ }^{3}$ Universidad Nacional de Educación a Distancia (Spain)
}

\begin{abstract}
This paper examines the association of cardiovascular reactivity with thermal thresholds (detection and unpleasantness). Heart period (HP), systolic (SBP) and diastolic (DBP) blood pressure of 42 health young males were recorded during a cardiovascular reactivity task (a videogame based upon Sidman's avoidance paradigm). Thermal sensitivity, assessing detection and unpleasantness thresholds with radiant heat in the forearm was also estimated for participants. Participants with differential scores in the cardiovascular variables from base line to task $\geq \mathrm{P}_{65}$ were considered as reactors and those how have differential scores $\leq \mathrm{P}_{35}$ were considered as non-reactors. Significant differences were observed between groups in the unpleasantness thresholds in blood pressure (BP) but not in HP. Reactors exhibited significant higher unpleasantness thresholds than non-reactors. No significant differences were obtained in detection thresholds between groups.

Keywords: cardiovascular reactivity, thermal sensitivity, unpleasantness threshold.
\end{abstract}

Este estudio investiga la relación entre la reactividad cardiovascular y la sensibilidad térmica (umbrales de detección y molestia). 42 varones jóvenes y sanos participaron en un experimento en el que se registraron el periodo cardiaco, la presión arterial sistólica y la presión arterial diastólica durante la realización de una tarea evocadora de reactividad cardiovascular (videojuego basado en el paradigma de evitación de Sidman). La sensibilidad térmica fue medida en todos los participantes mediante la estimación de los umbrales de detección y molestia en la piel del antebrazo con el método de calor radiante. Los participantes que presentaron valores diferenciales en las variables cardiovasculares respecto a la línea base $\geq P_{65}$ fueron considerados como reactores, mientras que los que obtuvieron valores diferenciales $\leq \mathrm{P}_{35}$ se consideraron como no-reactores.

Se encontraron diferencias significativas en los umbrales de molestia entre los grupos de reactores y no-reactores en presión arterial, aunque no se encontraron diferencias en la reactividad del periodo cardiaco. El grupo de reactores presenta umbrales más elevados en la molestia que el grupo de no-reactores. No se encontraron diferencias en los umbrales de detección entre los grupos. Palabras clave: reactividad cardiovascular, sensibilidad térmica, umbral de molestia.

Correspondence concerning this article should be addressed to Pablo Antonio Conde-Guzón. Área de Personalidad, Evaluación y Tratamiento Psicológicos. Departamento de Psicología, Sociología y Filosofía. Universidad de León. Campus de Vegazana s/n. 24071 León (Spain). Phone: +34-987782136. E-mail: pacong@unileon.es 
Arterial hypertension is a severe and common medical condition in nowadays developed countries, being one of the main cardiovascular risk factors. Is also known as the "silent killer" since people suffering it are not aware of any symptom or external signal. Nevertheless, an elevated blood pressure (BP) is usually accompanied by the following behavioural symptom: a reduction on pain sensitivity (hypoalgesia). This symptom, that could be considered as a benefit of the hypertension can, in fact, generate quite negative consequences on health. Epidemiological data from the Framingham Heart Study show that hypertensive patients double the possibilities of not recognising a heart attack than normotensive. This is, probably, a consequence of the lack of sensitivity to the sources of pain that usually come along with myocardial infarction (chest pain). One of the first studies that connected hypertension and hypoalgesia was conducted by Zamir and Segal (1979) with spontaneously hypertensive rats. These rats experienced a genetically predisposition to develop hypertension. During the $4^{\text {th }}$ week of life, they usually have normal BP levels, but by the beginning of the $12^{\text {th }}$ week, they have already developed hypertension. Hypoalgesia is commonly developed among them by the $3^{\text {rd }}$ week, indicating that this symptom precede the debut of hypertension and, therefore, could be related to the risk of developing hypertension rather than to established hypertension itself. Similar results were observed in humans afterwards. Hypertensive patients, in relation to normotensive, showed a reduction on pain thresholds when an electrical stimulation was inflicted on their tooth pulp (Ghione, Rosa, Mezzasalma, \& Panattoni, 1988).

As well as the hypoalgesia, elevated BP evoked by psychological stress has also being considered as a risk marker of hypertension or coronary diseases (Gerin et al., 2000; Light, Dolan, Davis, \& Sherwood, 1992; Moseley \& Linden, 2006; Steptoe \& Cropley, 2000; Stewart, Janicki, \& Kamarck, 2006). This is known as the reactivity hypothesis. For instance, Treiber et al. (2003) report three epidemiological studies with long term following up periods (up to 20 years) where BP evoked responses to cold pressor could be use as a predictor of the essential hypertension development among initially normotensive participants.

These hypertension risk factors (hypoalgesia and cardiovascular reactivity) are related each other: a reduction on pain sensitivity commonly appear on those individuals that exhibit an exaggerated cardiovascular reactivity evoked by stress (Burns, Bruehl, \& Caceres, 2004; Caceres \& Burns, 1997; Duschek, Schwarzkopf, \& Schandry, 2008; Edwards, et al., 2007; France, Froese, \& Stewart, 2002; Nyklícek, Vingerhoets, \& Van Heck, 1999). France and Ditto (1996) proposed a model that tried to explain this relation. This model, that extends McCubbin's proposals (1991), considers that there is failure on the hypothalamus normal regulation of the BP responses to stress and pain perception in hypertensive individuals. The reduction on pain perception associated to hypertension for this model is due to: 1) a greater increase in endogenous opioids during stress; 2) an increased stimulation of specific receptors involved in BP regulation; 3) and an enhanced stimulation of descending central nervous system pathways involved in pain modulation.

In the first case, a decreased sensitivity to endogenous opioids by certain hypothalamic neurons, results in a failure of normal regulating the responses to stress. This failure leads to an exaggerated BP increase as well as a greater release of endogenous opioids (e.g. $\beta$-endorphin from pituitary gland and enkephalins from adrenal medulla). Despite the additional release of endogenous opioids do not affect the BP levels, due to the hypothalamic insensitivity to opioids, it can contribute to a reduction on the pain sensitivity. This is as consequence of the fact that there are opioids receptors as well in the spinal cord.

In the second case, hypertensives cannot regulate their $\mathrm{BP}$ in the same extent as normotensives, because they exhibit a reduction in the baroreflex sensitivity. When BP increases, the sympathetic system do not reduce its activity and the parasympathetic system do not increases its activity, as it should. The sympathetic system remains active, making that BP levels not to decrease and to generate a greater release of enkephalins that could reduce the pain perception.

Finally, an extensive or prolonged inhibition of pain signals among hypertensives could be explained by the reduction of response to opioids that normally inhibits the hypothalamic activity along with a greater activation of the descendent pathways for modulating pain.

In most of the studies, where this decrease of pain perception associated to a greater cardiovascular reactivity has been described, procedures mainly have use pain thresholds. The use of cold pressor to generate pain is habitual among those studies (Burns, Bruehl, \& Caceres, 2004; Caceres \& Burns, 1997; Campbell, Holder, \& France, 2006; Myers, Robinson, Riley, \& Sheffield, 2001). Participants maintain their hands submerged in ice-cold water until they feel pain (pain threshold) or until they were not able to tolerate the pain (tolerance threshold). Paradoxically, the relation between cardiovascular reactivity and unpleasantness thresholds has been scarcely studied. With the aim of providing more evidences in this realm, the present study was carried out.

In our experiment the cardiovascular reactivity has been evoked by means of a videogame reaction time task, based upon the Sidman's avoidance paradigm. The pain sensitivity has been estimated from the non-nociceptive thermal thresholds by applying radiant heat on the skin of the forearm. In the estimation of these thresholds, latencies have been used as an index of thermal sensitivity. This method was elected because of its high reliability (Curio, 1990; Lee \& Stizer, 1995), low cost and simplicity in application. The use of latencies in order to estimate non-nociceptive thermal thresholds with radiant heat allow 
the use of lower temperatures that prevent tissular damage, thank to a central neural mechanism called "temporal summation" (Pertovaara, Kkauppila, \& Hhämäläinen, 1996). The procedure is also simple, because a small number of trials are needed for obtaining it. Two different thresholds have been recorded in our procedure: detection and unpleasantness. The first one does not imply any nociceptive consequences. The second could be considered in the boundaries of pain threshold. Therefore, we do not expect to find significant differences in the detection threshold between the group of participants that react with an exaggerated increase of BP during the stress task and those who do not. At the same time, we expect to find differences between those two groups in the unpleasantness thresholds.

\section{Method}

\section{Participants}

Fifty volunteer psychology students agreed to take part in this study. Participants were recruited from the university community by letters and advertisements. In the sample selection participant's medical status was taken into account. Following this criterion 42 subjects were selected. People in the next circumstances were excluded from sample: regular use of medication, diabetes mellitus, cerebrovascular diseases, myocardial infarction, angina, peripheral vascular diseases, neurological diseases, severe renal or hepatic dysfunctions, elevated alcohol consumption (over 28 units of alcohol per week), a past or present history of hypertension or a sever mental diseases. Individuals experiencing chronic pain were also discarded from the sample. Informed consent was obtained from all subjects. Sample characteristics appear in Table 1.

\section{Apparatus}

With the aim of measuring task evoked cardiovascular reactivity $\mathrm{EKG}$ and $\mathrm{BP}$ were continuously recorded during experimental session. EKG was recorded by means of a Bexen electrocardiograph (Bexline model). The electrode placement was the Standard Lead II configuration. BP was also recorded continuously with the Fin. A. Pres. Ohmeda 2300. This instrument record calibrated pulse wave at the finger, maximum corresponding to systolic pressure and minimum to diastolic. The physiological signals were inspected during the whole experiment. There are two different display devices that should be controlled: the oscilloscope for the EKG and the Fin. A. Pres. to monitor for blood pressure. All signals were stored digitally on a videotape, this require and analogical-digital converter (Neurocorder, model DR890). Conversion rate in both ways (analogical to digital or reverse), was one data per ms. None of the signal conversions implies neither any loss of information, nor phase shift. By storing signal into videotape, it is possible to check off-line if a given misrecording is due to an information loss or due to an artefact. The EKG was processed by computer software to detect R-waves, which indicate the peak of ventricular depolarization. The inter-beat interval was recorded. Once the EKG was analyzed the BP was processed, indicating the maximum (systolic) and minimum (diastolic) for each heart period. The missing data caused by the adjustments of the Fin. A. Pres. were estimated by an interpolation of a polynomic trend analysis to the power of five.

In order to assess the heat sensitivity, a specific device was developed by our research group (GESTER-01, international patent number: PCT/ES2005/000693). This device was based upon the "dolorimeter" designed by Hardy, Goodell and Wolff (1952). The GESTER-01 is composed of a parallelepiped shape box made of black metacrilate mounted on a stand. The height of the device was adjustable allowing the experimenter to adapt it to the physical characteristics of participants or to apply the stimulation on a different part of the body. Inside the box, there was a radiant source of heat with a built-in reflector $(\mathrm{a} 15 \mathrm{~V}$ and 150W Philips halogen lamp). To avoid overheat, internal temperature was kept constant by means of an electric fan. A console is used to control the levels of heat intensity (21 in total, ranging from $38.3 \mathrm{~W}$ to $136.3 \mathrm{~W}$ with successive

Table 1

Sample characteristics

\begin{tabular}{lrr}
\hline \multicolumn{1}{c}{ Variables } & Mean & Standard Deviation \\
\hline Age (years) & 26.9 & 2.02 \\
Body mass index $\left(\mathrm{kg} / \mathrm{m}^{2}\right)$ & 24.4 & 3.01 \\
Participants with a family history of cardiovascular disease $(N=15)$ & & $35.7 \%$ \\
Participants that regularly practice physical exercise $(N=24)$ & & $57.1 \%$ \\
Smokers $(N=28)$ & 120.1 & $66.7 \%$ \\
Baseline SBP $(\mathrm{mm} \mathrm{Hg})$ & 69.8 & 13.1 \\
Baseline DBP $(\mathrm{mm} \mathrm{Hg})$ & 828.6 & 6.2 \\
Baseline Heart period $(\mathrm{ms})$ & 80.3 \\
\hline
\end{tabular}


increments of $4.9 \mathrm{~W}$, calibrated in $\mathrm{mcal} / \mathrm{s} / \mathrm{cm}^{2}$ ), exposure times ( 0.1 to 0.99 seconds) and the intervals between stimuli ( 1 to 99 seconds). The power of the lamp was controlled by means of a variable transformer in order to minimize the effects caused by abrupt increases of temperature as a consequence of the caloric concentration (see Yarnistky \& Ochoa, 1990). The radian heat was applied by means of an elliptical mask on a $17.13 \mathrm{~cm}^{2}$ region of the forearm. The level of thermal energy used was of $16.6 \mathrm{mcal} / \mathrm{sec} /$ $\mathrm{cm}^{2}$ (consult Conde-Guzón et al., 2003, 2005, for a deeper explanation of the GESTER-01).

\section{Task and procedure}

All the participants performed two experimental sessions in order to assess independently the cardiovascular reactivity and the thermal thresholds of the heat sensitivity. Both sessions were counterbalanced between participants. All the session took place by morning time, approximately at the same hour. Between experimental sessions, a 15 minutes resting period was given to participants. The whole experiment lasted approximately one hour and a half. Prior to the experimental session, participants filled out a questionnaire about medical status, life style and personal data (age, height, weight, family history of cardiovascular diseases, practice of physical exercise, tobacco consumption, etc.). Once the questionnaire was completed participants were seated inside a sound attenuated and temperature controlled, Faraday's cage where the experiment took place.

Cardiovascular reactivity task: This task was composed by the following phases: an initial baseline (BL1) that lasted 5 minutes, a videogame that lasted 5 minutes and finally another baseline (BL2) of the same duration than the first one. Between the last two phases, a three minutes interval was introduced to study recovery.

After a habituation period of approximately 15 minutes when measures on the oscilloscope and the monitor of the Fin. A. Pres. were stable, the 5 minutes BL1 was recorded. The instructions given to the subjects for habituation and baseline were to stay as calm and steady as they can. Once BL1 was over, instructions for the task were provided. The task used to induce cardiovascular reactivity was a videogame reaction time task based on Sidman's avoidance paradigm (Sidman, 1953) ${ }^{1}$. It may be conceived as a complex operant, including two responses (r1-r2) that should be performed one after the other within a prefixed interval marked by means of a visual cue. Participants were presented a yellow background strip and two moving black lines coming from the edges to meet at the centre of the computer screen. Those lines appear by the time the participant call for the task pressing a keypad button (r1) and should be detained before they reach each other at the centre of the screen also by pressing another key ( $\mathrm{r} 2)$. The line speed and the temporal window (when the responses were considered as correct), could be programmed. Both parameters (as well as those imposed by Sidman's paradigm) served to determine task difficulty. Lines reach each other at the centre of the screen $720 \mathrm{~ms}$ after $\mathrm{rl}$, and the temporal window lasted $90 \mathrm{~ms}$. Therefore, $\mathrm{r} 2$ should be given between 630 and $720 \mathrm{~ms}$ to be considered as correct. Sidman's avoidance task requires two reinforcement schedules of variable interval: the shock-shock interval (S-S) and the response-shock interval (R-S). The SS interval was programmed within 3-5 seconds and was active while subject's response was not correct. If the interval reaches the end without a correct response by the subject, an aversive tone, as well as, a flashing red light was given. Also, one point was taken from those 10 given to the participant at the beginning of the task. Once a correct response occurs the R-S interval started lasting variably from 4 thru 8 seconds. After that period of time, the first correct response was reinforced (avoiding all the aversive consequences). If the R-S interval were not followed by a correct response $\mathrm{S}-\mathrm{S}$ interval became active. Otherwise, R-S interval starts again. All participants were instructed to keep as many points as the can.

Heat sensitivity assessment: During this phase, the detection and unpleasantness heat thresholds were determined for every participant. Prior to the heat sensitivity assessment task, the stimulation area was washed with neutral $\mathrm{pH}$ soap and blackened with Indian ink. Once the ink was dry, the assessment procedure started. For the detection heat thresholds, participants were instructed to press a button as soon as they began to feel the heat. For the unpleasantness thresholds, participants have to press the button as soon as they feel an unpleasant sensation. After these instructions, researcher commenced to apply the heat. The time lasted until the participant pressed the button was recorded. Several training trials were conducted to ensure participant comprehension. Twelve task trials were used, six for the detection threshold and other six for the unpleasantness one. They were assessed sequentially one after the other. Between every trial, a 3 minutes rest period was given to let the skin temperature come back to the initial values. During this period, Indian ink was again applied. A 5 minutes rest period was introduced between the assessments of both thresholds. Thresholds were calculated by obtaining the mean latencies of the six trials of the same condition.

\footnotetext{
1 It was designed in Max Planck Institute by Dr. Rupert Hölzl and it was handed over to our Department. The task was revised and reprogrammed by Dr. Grzib.
} 


\section{Results}

\section{Cardiovascular reactivity}

The arithmetic mean was calculated for every experimental condition and for each of the physiological variables recorded: heart period (HP), systolic (SBP) and diastolic (DBP) blood pressure. In order to check if the task has evoked cardiovascular reactivity, one-way repeated measures MANOVA was conducted between the three experimental conditions (BL1, Task, BL2) with all the cardiovascular variables (HP, SBP, DBP). A GeisserGreenhouse correction was used where the Mauchly $W$ led to rejection of the sphericity hypothesis; if so, epsilon $(\varepsilon)$ was reported. Bonferroni-adjusted $p$ values (SPSS) are provided for all pairwise comparisons. Values of $\eta_{p}{ }^{2}$ are also reported to provide information about the effect size.

Results indicated significant differences between the three experimental conditions and the linear combination of the cardiovascular variables: $\Lambda$ de Wilks $=.189 ; F(6,160)=$ $36.019 ; p<.001 ; \eta_{\mathrm{p}}{ }^{2}=.575$. Follow up univariate ANOVAs indicated a significant effect of all the cardiovascular variables. HP: $F(2,82)=14.31, p<.001, \varepsilon=.765, \eta_{\mathrm{p}}{ }^{2}=.259$; SBP: $F(2,82)=147.447, p<.001, \varepsilon=.709, \eta_{\mathrm{p}}{ }^{2}=.782$ and DBP: $F(2,82)=138.728, p<.001, \varepsilon=.799, \eta_{\mathrm{p}}{ }^{2}=.772$. Further post hoc analyses revealed significant differences between task and both BLs (Bonferroni adjusted, $p \mathrm{~s} \leq .001$ ). The HP was significant greater during the task $(M=781.097 \mathrm{~ms}$, $S D=113.219)$ than BL1 $(M=828.611 \mathrm{~ms}, S D=106.554)$ or BL2 $(M=824.051 \mathrm{~ms}, S D=110.662)$. Similarly, the SBP was significant higher during task $(M=165.206 \mathrm{~mm} \mathrm{Hg}$, $S D=21.829)$ than during BL1 $(M=135.094 \mathrm{~mm} \mathrm{Hg}, S D=$ $15.742)$ or BL2 $(M=140.830 \mathrm{~mm} \mathrm{Hg}, S D=16.823)$. Finally, the DBP react in a very similar manner than the SBP, significant higher values were obtained during task that during BLs (Task: $M=89.578 \mathrm{~mm} \mathrm{Hg}, S D=13.576$; BL1: $M=$ 75,939 mm Hg, $S D=12.913$; BL2: $M=79,960 \mathrm{~mm} \mathrm{Hg}$, $S D=12.451)$. Significant differences between BL1 and BL2 were also observed for SBP and DBP (Bonferroni adjusted, $p s<.001)$. This result indicates that the effects evoked by the task did not disappear completely during BL2.

\section{Relationship between sensitivity to heat and cardiovascular reactivity}

With the aim of classify participants in relation to cardiovascular reactivity, the values (HP, SBP or DBP) obtained in task were recalculated by subtracting the ones obtained during BL1. The percentiles 65 and 35 were used as a classification criterion. Participants with differential scores $\geq \mathrm{P}_{65}$ in the three cardiovascular variables were considered as reactors; and those how have differential scores $\leq \mathrm{P}_{35}$ were considered as non-reactors. This technique of assigning participants into different groups as a function of their reactivity has been commonly used by other researchers (Caceres \& Burns, 1997: France \& Stewart, 1995; Obrist, 1981). Taking this criterion into account, only 6 participants were considered as reactors and 4 as nonreactors for all the variables (HP, SBP and DBP). In order to increase the amount of participants in each of the groups, two different criterions were used to classify reactor and non-reactors: HP reactivity and BP reactivity (scores $\geq \mathrm{P}_{65}$ and scores $\leq \mathrm{P}_{35}$ for both variables $\mathrm{SBP}$ and $\mathrm{DBP}$ ). Following this HP classification criterion, 15 participants were considered as reactors and 15 as non-reactors. In the other hand, following the BP classification criterion, 12 participants were considered as reactors and 10 as nonreactors. Using non-parametric tests, those groups were compared in the following variables: family history of cardiovascular disease, practice of physical exercise, tobacco consumption. As well, a t-test was carried out to compare both groups in age and BMI. No significant differences were obtained in any of these variables.

To check if there were significant differences between reactors and non-reactors on a linear combination of the detection and unpleasantness thresholds, two one-way MANOVAs were calculated (one for each classification criterion: HP reactivity, BP reactivity).

Results indicated no significant differences between reactors and non-reactors in the linear combination of the detection and unpleasantness thresholds for HP reactivity. Nevertheless, significant differences were found for the BP reactivity: $\Lambda$ de Wilks $=.444, F(2,19)=11.889, p<$ $.001 ; \eta_{\mathrm{p}}^{2}=.556$. Follow up univariate ANOVAs showed significant differences between reactors and non-reactors in unpleasantness thresholds: $F(1,20)=22.885, p<.001$; $\eta_{\mathrm{p}}{ }^{2}=.534$. Reactors group showed unpleasantness thresholds higher than non-reactors group. On the other hand, in detection thresholds, similar results were found for both groups. Detection and unpleasantness thresholds for all the cardiovascular variables can be seen in figure 1 .

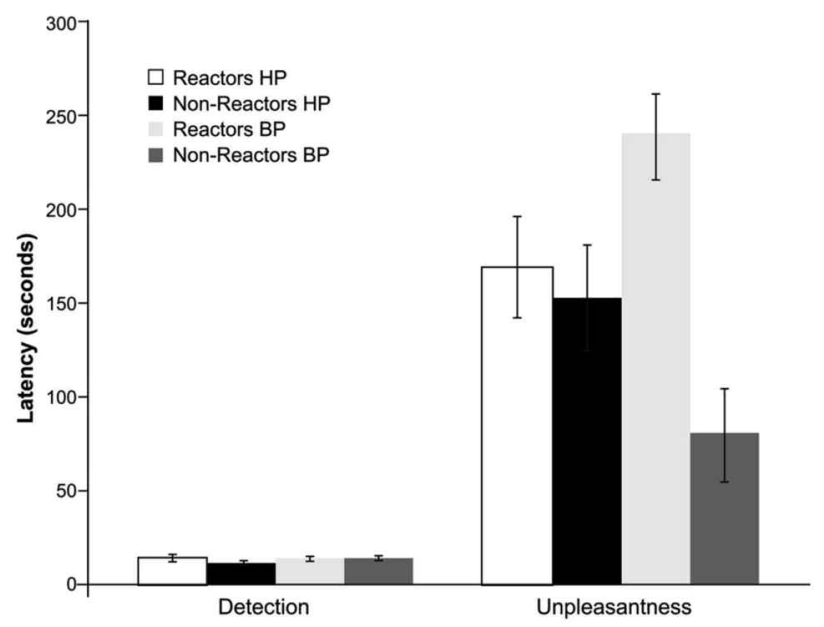

Figure 1. Detection and Unpleasantness thresholds as a function of different cardiovascular reactivity. 
Additionally, several one-way MANOVAs were calculated in order to check if participants with higher risks of developing hypertension in the future (those with family history of cardiovascular disease, tobacco consumption and sedentarism; in comparison with those who were not presenting this risk), were behaving in a similar way than participants who showed a higher cardiovascular reactivity in BP. Those analyses were performed with the whole sample checking each risk factor at a turn. The results indicated only significant differences in the linear combination of the detection and unpleasantness thresholds in relation to the tobacco consumption: $\Lambda$ de Wilks $=.832, F(2,39)=3.932, p=.028, \eta_{\mathrm{p}}{ }^{2}=.168$. Follow up univariate ANOVA showed that smokers exhibited higher unpleasantness thresholds than no-smokers: $F(1,41)=7.828$, $p=.008, \eta_{\mathrm{p}}^{2}=.164$. Unpleasantness and detection thresholds are shown in figure 2. Also, as it can be observed in figure 2 , participants with a family history of cardiovascular disease had higher unpleasantness thresholds, though this differences were not statistical significant.

In order to compare if smokers and non-smokers exhibited different values of cardiovascular reactivity, a mixed MANOVA was calculated between the three experimental conditions (BL1, Task, BL2) with all the cardiovascular variables (HP, SBP, DBP) for both groups. Box's $M$ test was not significant, therefore homogeneity of covariance matrices can be assumed. Significant multivariate effects were found for the main effects of the group: $F(3,38)=4.294, p=.011, \eta_{\mathrm{p}}{ }^{2}=.253$, as well as for the interaction between groups and reactivity: $F(6,35)$ $=3.446, p=.009, \eta_{\mathrm{p}}{ }^{2}=.371$. Follow up ANOVAs revealed that the interaction effect was only significant for the SBP: $F(2,80)=4.533, \varepsilon=.696, p=.026 ; \eta_{\mathrm{p}}^{2}=.102$. Further post hoc analyses revealed significant differences between smokers and non-smokers in the three experimental conditions (Bonferroni adjusted, $p \mathrm{~s} \leq .001$ ). Smokers, when compared to non-smokers, exhibited higher SBP values in BL1, reacted in a bigger extent during the task and, also, had a worst recovery at BL2, as shown in Table 2.

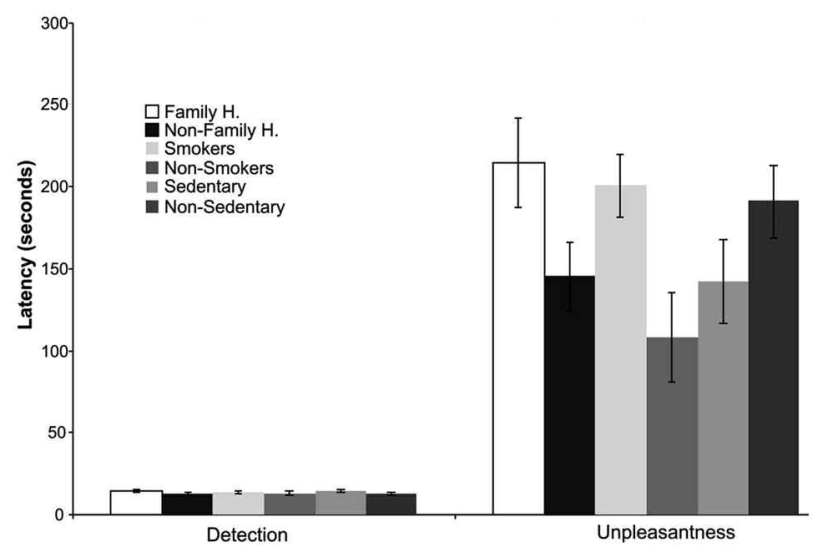

Figure 2. Detection and Unpleasantness thresholds as a function of different cardiovascular risk factors.
Table 1

Mean and Standard Deviation of SBP by condition as a function of tobacco consumption

\begin{tabular}{lccc}
\hline & \multicolumn{3}{c}{ SBP (mm Hg) } \\
\cline { 2 - 4 } & BL1 & Task & BL2 \\
\hline Smokers & 138.69 & 172.59 & 146.64 \\
$(n=28)$ & $(14.92)$ & $(17.61)$ & $(14.98)$ \\
Non-smokers & 127.89 & 150.43 & 129.21 \\
$(n=14)$ & $(15.33)$ & $(22.50)$ & $(14.43)$ \\
\hline
\end{tabular}

\section{Discussion}

The major finding of this study is the confirmation that healthy male with greater blood pressure reactivity, during a videogame reaction time task, exhibit significant higher unpleasantness thermal thresholds than non-reactors. A significant increase in the unpleasantness threshold measured on forearm was found in participants showing greater reactivity in blood pressure. However, they did not differ in the detection thresholds. On the other hand, as a result of our data, no relations could be established between heart period reactivity and any of the thresholds (detection or unpleasantness). This fact reinforces the idea that is cardiovascular reactivity in BP, the one that is mainly related with the increase of sensitivity to unpleasantness stimulation. Baroreceptors could be considered as playing part of a shared physiological modulatory mechanism. In agreement with the results noted here, other authors (Parker, et al., 1987; Menkes, et al., 1989) likewise suggest that reactivity in blood pressure is a better predictor of the relationship between sensitivity to unpleasantness stimulation and the development of hypertension, rather than to reactivity in heart rate.

Additionally, we have also found that smokers exhibited higher unpleasantness thresholds that non-smokers. This result has been found in previous studies. For instance, Girdler et al., (2005) affirm that the reduced pain perception in smokers is a result of a dysregulation in endogenous pain mechanisms. In this sense, experimental data bring evidence for analgesic action of nicotine acting via nicotinic acetylcholine receptors. Smokers take much more analgesics than non-smokers and probability of developing opioid dependence is increased in this group of patients. Some studies demonstrate increased requirements for postoperative opioid analgesia in smoking patients (Billert, Gaca, \& Adamski, 2007; Shaw \& al'Absi, 2010).

As outlined in the introduction section, the relationship between hypoalgesia and cardiovascular reactivity may be mediated by any of the following three basic mechanisms modulated by the hypothalamus (paraventricular nucleus): baroreceptor reflex arc stimulation, endogenous opioid responsivity, and descending pain-modulating pathways 
(Bruehl \& Chung, 2006; France \& Ditto, 1996; McCubbin, 1991). The results obtained could support this proposal. These authors suggest that the paraventricular nucleus of the hypothalamus stops responding normally to the action of endogenous opiates in individuals in risk of developing arterial hypertension (e.g. cardiovascular reactors in stress situations). Interestingly, our research extends the relationship, between hypoalgesia and cardiovascular reactivity, to the unpleasantness thresholds. Such association can only be ensured if the cardiovascular system shares some anatomic and functional pathways with the pain sensation.

Taking all above reflections into account, it may be held that the results obtained for sensitivity to unpleasantness on the forearm can be considered as a good marker for cardiovascular reactivity in arterial pressure, and hence for a future risk of developing hypertension. To put it in another way, the appearance of signs of a higher threshold of unpleasantness might precede the development of arterial hypertension, because cardiovascular reactivity constitutes a precursor of high arterial blood pressure.

In order to generalize these results, some considerations for future work should be taken into account: First, to increase the sample size and, Secondly, to include a female sample for studying the influence of the gender in these results, Third, to use different tasks to generate stress.

Finally, this study simply intended to provide further evidences of lowered sensitivity among people with normal blood pressure but who appear to be at risk of developing arterial hypertension (as showing higher level of cardiovascular reactivity to stress in the laboratory). All above results open a new trail for future research that would cover in dept the physiological and anatomical correlates of the relationship between cardiovascular reactivity under stress situations and pain sensitivity.

\section{References}

Billert, H., Gaca, M., \& Adamski, D. (2007). Smoking cigarettes and pain-implications for the postoperative period. Przegl Lek, 64, 882-885.

Bruehl, S., \& Chung, O. Y. (2006). Parental history of chronic pain may be associated with impairments in endogenous opioid analgesic systems. Pain, 124, 287-294. doi:10.1016/j.pain. 2006.04.018

Burns, J. W., Bruehl, S., \& Caceres, C. (2004). Anger management style, blood pressure reactivity, and acute pain sensitivity: evidence for "Trait x Situation" models. Annals of Behavioral Medicine, 27, 195-204. doi:10.1207/s15324796abm2703_7

Caceres, C., \& Burns J. W. (1997). Cardiovascular reactivity to psychological stress may enhance subsequent pain sensitivity. Pain, 69, 237-244. doi:10.1016/S0304-3959(96)03289-7

Campbell, T. S., Holder, M. D., \& France, C. R. (2006). The effects of experimenter status and cardiovascular reactivity on pain reports. Pain, 125, 264-269. doi:10.1016/j.pain.2006.06.002
Conde-Guzón, P. A., Bartolomé-Albistegui, M. T., Quirós-Expósito, P., \& Grzib Schlosky. (2003). Hipertensión, reactividad cardiovascular ante el estrés y sensibilidad al dolor [Hypertension, cardiovascular reactivity to stress, and pain sensibility]. Revista de Neurología, 37(6), 586-595.

Conde-Guzón, P. A., Bartolomé-Albistegui, M. T., Quirós, P., Grzib, G., Cabestrero, R., \& Crespo, A. (2005). Assessing pain thresholds by applying radiant heat to the skin. Psychologica, 40, 213-230.

Curio, I. (1990). Reliability of thermal pain thresholds. Psychologische Beiträge 32, 16-22.

Duschek, S., Schwarzkopf, W., \& Schandry, R. (2008). Increased pain sensitivity in low blood pressure. Journal of Psychophysiology, 22(1), 20-27. doi:10.1027/02698803. 22.1.20

Edwards, L., Ring, C., France, C. R., al'Absi, M., McIntyre, D., Carroll, D., \& Martin, U. (2007). Nociceptive flexion reflex thresholds and pain during rest and computer game play in patients with hypertension and individuals at risk for hypertension. Biological Psychology, 76, 72-82. doi:10.1016/j. biopsycho.2007.06.005

France, C. R., \& Stewart, K. (1995). Parental history of hypertension and enhanced cardiovascular reactivity are associated with decreased pain ratings. Psychophysiology, 32, 571-578. doi:10.1037/0278-6133.17.3.249

France, C. R., \& Ditto, B. (1996). Risk for high blood pressure and decreased pain perception. Current Directions in Psychological Science, 5, 120-125. doi:10.1111/14678721.ep11452781

France, C. R., Froese, S. A., \& Stewart, J. C. (2002). Altered central nervous system processing of noxious stimuli contributes to decreased nociceptive responding in individuals at risk for hypertension. Pain, 98, 101-108. doi:10.1016/S03043959(01)00477-8

Gerin, W., Pickering, T. G., Glynn, L., Christenfeld, N., Schwartz, A., Carroll, D., \& Davidson, K. (2000). An historic context for behavioral models of hypertension. Journal of Psychosomatic Research, 48(4), 369-377. doi:10.1016/S0022-3999(99)00095-1

Ghione, S., Rosa, C., Mezzasalma, L., \& Panattoni, E. (1988). Arterial hypertension is associated with hypalgesia in humans. Hypertension, 12(5), 491-497.

Girdler, S. S., Maixner, W., Naftel, H. A., Stewart, P. W., Moretz, R. L., \& Light, K. C. (2005). Cigarette smoking, stress-induced analgesia and pain perception in men and women. Pain, 114(3), 372-385. doi:10.1016/j.pain.2004.12.035

Hardy, J. D., Goodell, H., \& Wolff, H. G. (1952). Pain sensations and reactions. Baltimore, MD: Williams and Wilkins

Lee, J. H., \& Stitzer, M. L. (1995). A novel radiant heat test for assessing pain threshold in human subjects. Measurement stability. Behavior Research Methods, Instruments \& Computers, 27, 41-45.

Light, K. C., Dolan, C. A., Davis, M. R., \& Sherwood, A. (1992). Cardiovascular responses to an active coping challenge as predictors of blood pressure patters 10 to 15 years later. Psychosomatic Medicine, 54, 217-230. 
McCubbin, J. A. (1991). Diminished opioid inhibition of blood pressure and pituitary function in hypertension development. In J. A. McCubbin, P. G. Kaufmann, \& C. B. Nemeroff (Eds.), Stress, Neuropeptides, and Systemic disease (pp. 445-466). San Diego, CA: Academic Press.

Menkes, M. S., Matthews, K. A., Krantz, D. S., Lundberg, W., Mead, L. A., Qaqish, B.,... Pearson, T. A. (1989). Cardiovascular reactivity to the cold pressor test as predictor of hypertension. Hypertension, 14, 524-530.

Moseley, J. V., \& Linden, W. (2006). Predicting blood pressure and heart rate change with cardiovascular reactivity and recovery: results from 3-year and 10-year follow up. Psychosomatic Medicine, 68, 833-843. doi:10.1097/01. psy.0000238453.11324.d5

Myers, C. D., Robinson, M. E., Riley, J. L., III, \& Sheffield, D. (2001). Sex, gender, and blood pressure: Contributions to experimental pain report. Psychosomatic Medicine, 63(4), 545-550.

Nyklícek, I., Vingerhoets, A. J. J. M., \& Van Heck, G. L. (1999). Hypertension and pain sensitivity: Effects of gender and cardiovascular reactivity. Biological Psychology, 50(2), 127142. doi:10.1016/S0301-0511(99)00006-X

Obrist, P.A. (1981). Cardiovascular psychophysiology: A perspective. New York, NY: Plenum.

Parker, F., Croft, J., Cresanta, J., Freedman, D., Burke, G., Webber, L., \& Berenson, G. (1987). The association between cardivascular response tasks and future blood pressure levels in children: Bogalusa Heart Studi. American Heart Journal, 113, 1174-1179. doi:10.1016/0002-8703(87)90931-8

Pertovaara, A., Kauppila, T., \& Hämäläinen, M. (1996). Influence of skin temperature on heat pain threshold in humans. Experimental Brain Research, 107(3), 497-503. doi:10.1007/ BF00230429
Shaw D., \& al'Absi, M. (2010). Blunted opiate modulation of prolactin response in smoking men and women. Pharmacology, Biochemistry and Behavior, 95, 1-5. doi:10.1016/j.pbb.2009. 11.016

Sidman, M. (1953). Avoidance conditioning with brief shock and no exteroceptive warning signal. Science, 118, 157-158. doi:10.1126/science.118.3058.157

Steptoe, A., \& Cropley, M. (2000). Persistent high job demands and reactivity to mental predict future ambulatory blood pressure. Journal of Hypertension, 18, 581-586. doi:10. 1097/00004872-200018050-00011

Stewart, J. C., Janicki, D. L., \& Kamarck, T. W. (2006). Cardiovascular reactivity to and recovery from psychological challenge as predictors of 3-year change in blood pressure. Health Psychology, 25, 111-118. doi:10.1037/02786133.25.1.111

Treiber, F. A., Kamarck, T., Schneiderman, N., Sheffield, D., Kapuku, G., \& Taylor, T. (2003). Cardiovascular reactivity and development of preclinical and clinical disease states. Psychosomatic Medicine, 65(1), 46-62.

Yarnitsky, D., \& Ochoa, J. L. (1990). Studies of heat pain sensation in man: perception thresholds, rate of stimulus rise and reaction time. Pain, 40(1), 85-91. doi:10.1016/03043959(90) 91055-N

Zamir, N., \& Segal, M. (1979). Hypertension-induced analgesia: Changes in pain sensitivity in experimental hypertensive rats. Brain Research, 160(1), 170-173. doi:10.1016/00068993(79)90614-0

Received February 28, 2008 Revision received September 8, 2010 Accepted October 20, 2010 